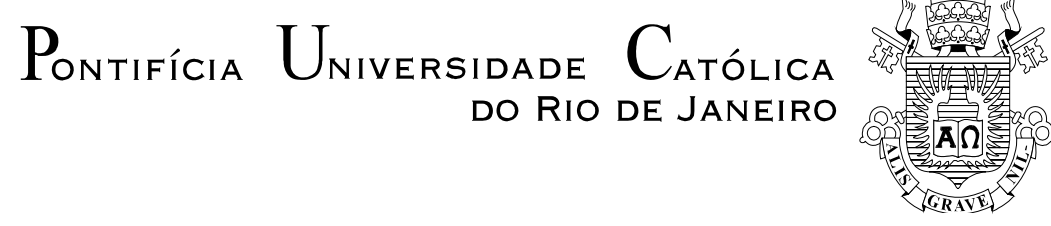

Rudy Massami Sakamoto Kawabata

Produção e Caracterização de Fotodetectores de Infravermelho baseado em Poços Quânticos com duas Geometrias Distintas de Acoplamento com a Luz

Dissertação de Mestrado

Dissertação apresentada ao Programa de Pós-Graduação em Engenharia Elétrica da PUC-Rio como requisito parcial para obtenção do título de Mestre em Engenharia Elétrica.

Orientadora: Profa. Patrícia Lustoza de Souza

Rio de Janeiro

Agosto de 2011 


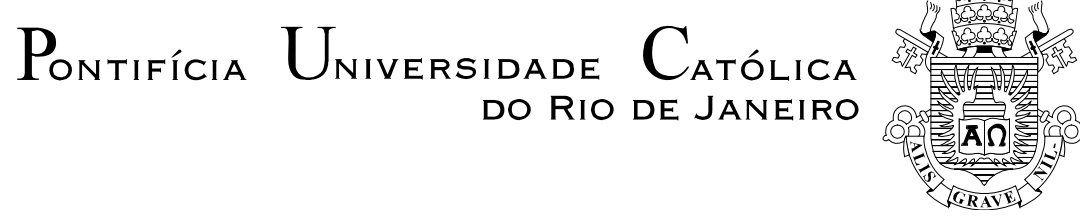

Rudy Massami Sakamoto Kawabata

\section{Produção e Caracterização de Fotodetectores de Infravermelho baseado em Poços Quânticos com duas Geometrias Distintas de Acoplamento com a Luz}

Dissertação de Mestrado apresentada como requisito parcial para obtenção do grau de Mestre pelo Programa de Pós-Graduação em Engenharia Elétrica do Departamento de Engenharia Elétrica do Centro Técnico Científico da PUC-Rio. Aprovada pela Comissão Examinadora abaixo assinada.

Profa. Patrícia Lustoza de Souza

Orientadora

Centro de Estudos em Telecomunicações - PUC-Rio

Profa. Luciene da Silva Demenicis

IME

Prof. Newton Cesário Frateschi

UNICAMP

Prof. José Eugenio Leal

Coordenador Setorial do

Centro Técnico Científico - PUC-Rio

Rio de Janeiro, 24 de agosto de 2011 
Todos os direitos reservados. É proibida a reprodução total ou parcial do trabalho sem autorização da universidade, do autor e da orientadora.

\section{Rudy Massami Sakamoto Kawabata}

Graduação em Bacharelado em Física pela Universidade Federal do Rio de Janeiro (UFRJ) em 2007. Ocupou a função de técnico especialista para o Laboratório de Semicondutores na Pontifícia Universidade Católica do Rio de Janeiro (PUC-Rio) de Março de 2010 a Julho de 2011.

Ficha Catalográfica

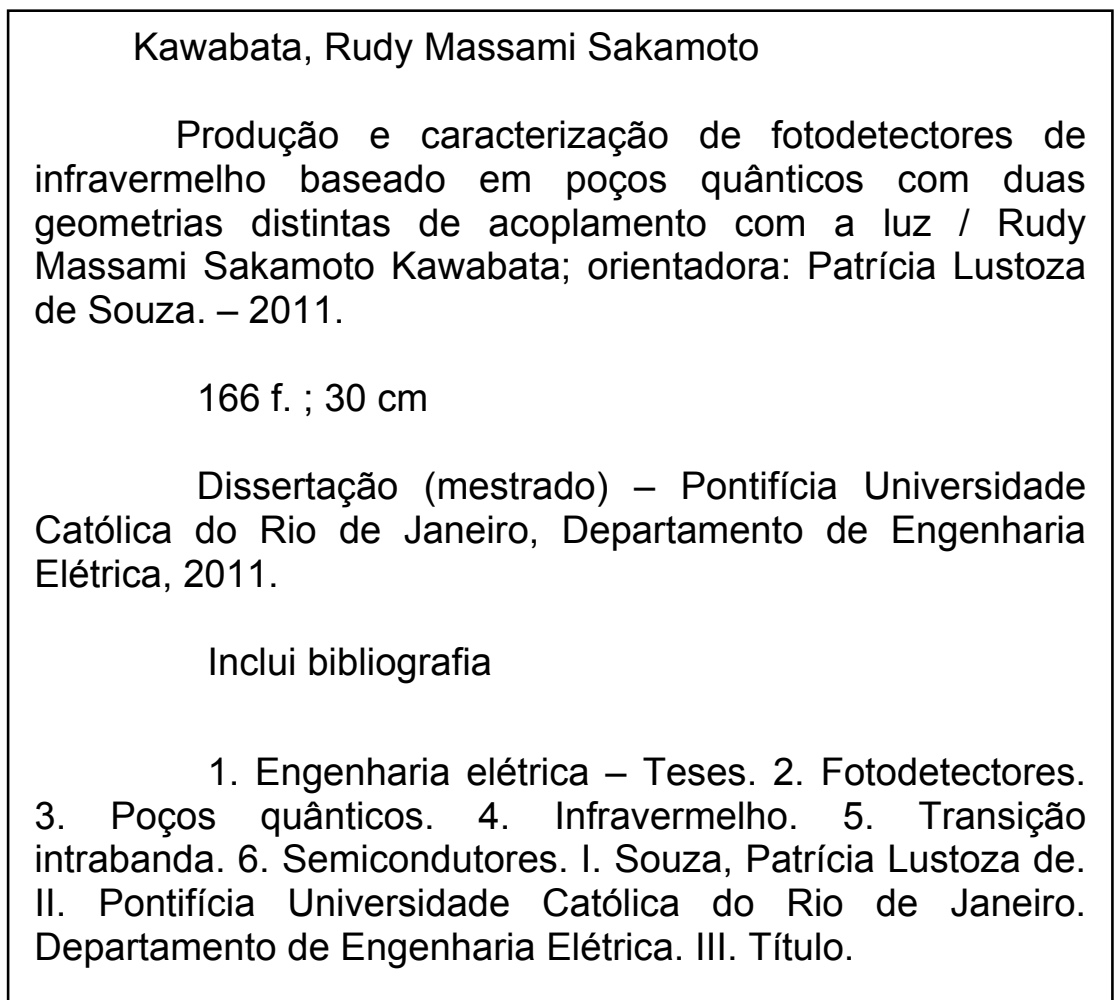


- いやねえ、世の中って

一 そう。嫌なことばっかり

(Tokyo Monogatari, 1953.

Yasujiro Ozu) 


\section{Agradecimentos}

Primeiramente à minha família pelo suporte em todos os sentidos. São o meu porto seguro sempre. Nominalmente meus pais Lucas Ikumi Kawabata e Neide Miyako Sakamoto Kawabata, além do meu avô Yutaka Sakamoto.

À orientadora Patrícia Lustoza de Souza pelo meu acolhimento como orientando, pelos conhecimentos passados em reuniões, correções sobre os diversos trabalhos realizados e o tratamento sempre gentil e resoluto não só comigo, mas com todos.

Ao co-orientador Mauricio Pamplona Pires, da UFRJ, pela indicação ao laboratório, aos ensinamentos práticos laboratoriais sem a qual não seria possível a conclusão da dissertação e o senso de humor sempre ativo pra alegrar o ambiente.

Ao técnico e químico Iracildo pela cotidiana briga contra todos os pepinos que surgiam no laboratório, pela grande ajuda nos assuntos relacionados ao manuseio de produtos químicos, aos ensinamentos sobre segurança no laboratório e às discussões sobre a vida profissional.

Aos dois colegas de estudo provenientes desde a graduação na UFRJ, ambos físicos: Germano Maioli Penello e Daniel Neves Micha. Pelas discussões teóricas, ajudas em medições experimentais, companheiros de congressos, piadistas nas horas vagas e ocupadas. Sem eles nenhum trabalho poderia ter sido concluído.

Aos alunos LabSemzísticos que passaram e deixaram suas marcas, seja ajudando ou atrapalhando. Dos mais antigos aos mais frescos: Teo Camarotti, Anna Marin, Alan Maioli, Luiza Schulz e Guilherme Torelly. Aos alunos estrangeiros que também realizaram trabalhos relevantes à minha dissertação: Larbi, Stefan Dornstauder, Michael Rockus e Aurienne Olivier. E aos alunos do IME que agregaram resultados importantes ao processamento: Felipe Santiago, Renê Timbó e Lucas Cunha.

Ao Gustavo Vieira, do Centro Técnico Aeroespacial, e a Marcela Freitas pela grande ajuda nas medições em São José dos Campos e por se mostrarem sempre muito prestativos quando eu necessitei.

Ao prof. Wagner Nunes Rodrigues e seus alunos que produziram uma das etapas mais críticas do processamento e contribuíram expandindo o objetivo inicial com idéias e ações interessantes.

Ao Dr. Karl Unterrainer e Tomas Gebhard, da Technical University Vienna, pela produção de uma das etapas da dissertação que não seria possível em nosso país no momento.

Ao professor Ivan Guillermo Solorzano, os alunos Martin Mendoza e Geronimo Perez, da Engenharia de Materiais, por terem cedido a utilização de seu laboratório em uma das etapas de produção das amostras.

Ao Marcio Biasoli e ao Giuliano Maiolini do Centro de Tecnologia da Informação (CTI) pelo apoio quanto a produção, corte e solda dos dispositivos. 
Aos colegas do grupo INCT DISSE que contribuíram à dissertação com conselhos, ajudas e ensinamentos: Anderson Singulani, Paulo Sergio Soares Guimarães, José Maria Villas-Boas, entre outros.

À PUC-Rio por ter me proporcionado realizar o mestrado com bolsa e pela compreensão na prorrogação de defesa.

E a todos que se julguem ter ajudado e que no momento me escapa da memória, minhas desculpas. 


\section{Resumo}

Kawabata, Rudy Massami Sakamoto; Souza, Patrícia Lustoza de (Orientadora). Produção e caracterização de fotodetectores de infravermelho baseado em poços quânticos com duas geometrias distintas de acoplamento com a luz. Rio de Janeiro, 2011. 166p. Dissertação de Mestrado - Departamento de Engenharia Elétrica, Pontifícia Universidade Católica do Rio de Janeiro.

Detectores de infravermelho possuem larga gama de aplicações em diversos setores, desde militares (visão noturna, mísseis) até civis (aparelhos eletrônicos). Nesta dissertação estivemos interessados nas absorções intrabanda de heteroestruturas multiepitaxiais com intuito de absorver infravermelho em 4,1 micra onde se localiza a primeira janela de transmissão atmosférica. Baseamos nossas heteroestruturas de poços quânticos em semicondutores da família III-V. Discorremos quanto a produção do dispositivo de forma detalhada, juntamente com todos os processos de calibração de cada etapa. O crescimento se dá pela técnica de MOVPE que possui alta precisão em termos da espessura e composição da camada depositada. Em seguida discutimos sobre o processamento da amostra crescida para expor os contatos elétricos. E finalizamos descrevendo o processo de integração do dispositivo sobre um suporte para leitura do sinal. Finalizada a etapa de produção, fizemos um estudo quanto às características da amostra tanto qualitativamente quanto quantitativamente. Este estudo objetivou a obtenção de duas informações: comparação direta entre as geometrias de acoplamento luminoso; e medição da eficiência dos detectores produzidos. Ao fim do trabalho obtivemos um fotodetector produzido desde seu crescimento até sua montagem final. Assim como os resultados da eficiência dos mesmos que já indicaram melhorias possíveis para trabalhos futuros. Visando a formação de um mercado de produção em larga escala de fotodetectores, este trabalho identificou áreas com carência de técnicas disponíveis e que necessitam de investimento.

\section{Palavras-chave}

Engenharia elétrica - Teses; fotodetectores; poços quânticos; infravermelho; transição intrabanda; semicondutores. 


\section{Abstract}

Kawabata, Rudy Massami Sakamoto; Souza, Patrícia Lustoza de (Advisor). Production and characterization of infrared photodetectors based on quantum wells with two distinct geometries for light coupling. Rio de Janeiro, 2011. 166p. MSc Dissertation - Departamento de Engenharia Elétrica, Pontifícia Universidade Católica do Rio de Janeiro.

Infrared detectors have a wide range of applications in various industries, from military (night vision, missile) to civil (electronics). In this dissertation we were interested in the intraband absorption of multiepitaxial heterostructures with aim at absorption of 4.1 microns infrared where there's located the first atmospheric transmission window. We based our quantum well heterostructures in semiconductor from the III-V family. We discourse about the production of the device in detail, along with all the calibration procedures for each step. The growth is done by MOVPE technique that has high accuracy in terms of thickness and composition of the deposited layer. We then discuss about the processing of the grown sample to expose the electrical contacts. And finally we describe the process of integration of the device over a base for reading the signal. Completed the production stage, we studied the characteristics of the sample both qualitatively and quantitatively. This study aimed to obtain two pieces of information: a direct comparison between the methods for light coupling, and measuring the efficiency of the detectors produced. At the end of the work we obtained a photodetector produced from its growth till its final assembly. Also we obtained the results of the efficiency of the sample that already indicated possible improvements for future works. If the aim is at the formation of a large-scale production of photodetectors, this study identified areas with a shortage of available techniques and in need of investment.

\section{Keywords}

Electrical engineering - Thesis; photodetectors; quantum wells; infrared; intraband transition; semiconductors. 


\section{Sumário}

1. Introdução 23

1.1. Motivação 23

1.2. Aplicações de radiação não-visível 24

$\begin{array}{ll}\text { 1.3. Fotodetectores de infravermelho } & 27\end{array}$

1.4. Imageamento térmico $\quad 30$

1.5. Objetivo da dissertação 34

2. Conceitos Teóricos 36

2.1. Poço quântico 36

2.1.1. Introdução 36

2.1.2. Poço quântico com barreira infinita 37

2.1.3. Poço quântico com barreira finita 39

2.2. Fotodetector de infravermelho baseado em poços quânticos 43

2.2.1. Conformação do poço quântico $\quad 43$

2.2.2. Distribuição de portadores $\quad 45$

2.2.3. Dispositivo por transições intrabanda 49

2.2.4. Acoplamento com a radiação eletromagnética 53

2.3. Grade de difração $\quad 55$

2.4. Corpo negro e transferência de fluxo $\quad 57$

2.5. Figuras de mérito $\quad 61$

2.5.1. Responsividade $\quad 61$

2.5.2. Detectividade 64

3. Produção e técnicas de caracterização 66

$\begin{array}{ll}\text { 3.1. Crescimento epitaxial } & 67 \\ & 67\end{array}$

3.1.1. Deposição por MOVPE 67

3.1.2. Caracterização por difração de raios-X $\quad 74$

3.1.3. Caracterização por fotoluminescência $\quad 79$

3.1.4. Caracterização por medida de efeito Hall 82

3.2. Processamento do fotodetector 85

$\begin{array}{ll}\text { 3.2.1. Fotolitografia } & 87\end{array}$

3.2.2. Corrosão das mesas $\quad 88$

3.2.3. Metalização dos terminais elétricos 90

3.2.4. Polimento em ângulo 93

3.2.5. Concepção da grade de difração $\quad 95$

3.2.6. Polimento na superfície inferior $\quad 97$

3.3. Montagem do fotodetector 98

3.3.1. Passivação $\quad 99$

3.3.2. Separação dos fotodetectores $\quad 101$

3.3.3. Solda sobre o suporte 102

3.4. Técnicas de caracterização do fotodetector 104

3.4.1. Corrente de escuro 105

3.4.2. Ruído 108

3.4.3. Espectro de fotocorrente 109 
3.4.4. Responsividade

4. Resultados e discussões

4.1. Produção

4.1.1. Crescimento

4.1.2. Processamento

126

4.1.3. Montagem

4.2. Caracterização

4.2.1. Corrente de escuro

4.2.2. Espectro de Fotocorrente

4.2.3. Responsividade

4.2.4. Detectividade 


\section{Lista de figuras}

Figura 1.1 - Aplicações cotidianas para radiação fora da faixa do visível ao olho humano.

Figura 1.2 - Aplicações técnicas para radiação fora da faixa do visível ao olho humano.

Figura 1.3 - Representação gráfica do espectro eletromagnético com as nomenclaturas comumente utilizadas para classificação de cada faixa.

Figura 1.4 - Representação gráfica do funcionamento de um sistema de detecção de gases [2] e um sistema de transferência de radiação infravermelha emitida por um laser de infravermelho através de uma fibra ótica e captada por um detector de infravermelho.

Figura 1.5 - Resultado do cálculo para a estrutura de bandas do silício cristalino.

Figura 1.6 - Representação simplificada para estrutura de bandas apresentada na figura 1.5.

Figura 1.7 - Os casos possíveis de preenchimentos da banda eletrônica de um sólido.

Figura 1.8 - Esquema de promoção de um elétron da banda de valência à banda de condução através da absorção de um fóton com energia igual à $E_{g}$.

Figura 1.9 - Imagem de uma câmera com detecção em 10 micra em uma cena com a presença de um recipiente com água gelada e outro com água quente. Nota-se a diferença de tonalidade referente a temperatura da água.

Figura 1.10 - Gráfico da transmissão de radiação pela atmosfera em função do comprimento de onda da radiação em questão. Há duas janelas de maior interesse entre 3 e 5 micra, e entre 8 e 14 micra. 
Figura 2.1 - Representação gráfica do poço de potencial infinito limitado por $-\mathrm{L} / 2$ e $\mathrm{L} / 2$, e as soluções discretas para as energias permitidas para uma partícula confinada dentro deste poço.

Figura 2.2 - Representação gráfica de um poço de potencial com barreiras finitas. Inclui-se as duas condições possíveis de energia de uma partícula neste sistema.

Figura 2.3 - Solução gráfica para as equações transcendentais apresentadas em 2.13 e 2.14 . Repare que temos números restritos de soluções possíveis e que dependendo dos parâmetros usados podemos ter apenas uma solução possível de nível confinado dentro do poço.

Figura 2.4 - Representação gráfica de uma barreira de potencial de espessura finita e uma solução de função de onda de uma partícula com energia abaixo da barreira.

Figura 2.5 - Formação de um poço de potencial utilizando-se 2 semicondutores com gaps distintos.

Figura 2.6 - Representação da formação de um poço quântico com 2 níveis discretos. $\mathrm{Na}$ banda de condução temos níveis para elétrons e na banda de valência temos níveis para buracos.

Figura 2.7 - Gráfico da densidade de estados disponíveis para elétrons confinados em diversos sistemas.

Figura 2.8 - Esquemático de uma estrutura de GaAs com uma impureza de Si inserido na rede cristalino no lugar de um átomo de gálio. Esta troca introduz um elétron a mais no sistema.

Figura 2.9 - Representação da dopagem do poço na esquematização da estrutura de bandas de uma heteroestrutura.

Figura 2.10 - Representação de um fotodetector do tipo bound to continuum.

Figura 2.11 - Representação de um fotodetector do tipo bound to bound.

Figura 2.12 - Representação de um fotodetector do tipo bound to quasibound. 
Figura 2.13 - Esquemático de três possibilidades de incidência de radiação sobre uma amostra de poços quânticos. A primeira referente a uma incidência convencional mas que não renderá boa absorção, e as demais revelando as duas propostas sugeridas para acoplamento da radiação incidente.

Figura 2.14 - Esquematização de uma grade de difração com dois feixes monocromáticos incidentes. Para que a interferência entre os dois feixes seja construtiva, temos que (dsenq+dsenq) devem ser um múltiplo inteiro do comprimento de onda do feixe.

Figura 2.15 - Esquematização da grade de difração retangular utilizada nos fotodetectores heteroestruturados com poços quânticos.

Figura 2.16 - Sistema de coordenadas esféricas.

Figura 2.17 - Emissão de corpo negro para algumas temperaturas para a faixa de comprimentos de onda em MWIR e LWIR. Fonte: www.lprl.org

Figura 2.18 - Transferência de fluxo do corpo negro de abertura $\mathrm{A}_{\mathrm{s}}$ até o detector de área $A_{d}$.

Figura 2.19 - Representação da modulação da abertura do corpo negro $\left(\mathrm{A}_{\mathrm{s}}\right)$ por um modulador (chopper). [20]

Figura 2.20 - Fator de transferência em função de c.

Figura 2.21 - Exemplo de um espectro de ruído em função da frequência. Nota-se a presença da contribuição do ruído $1 / \mathrm{f}$ em vermelho e a contribuição do ruído branco em azul.

Figura 3.1 - Organograma da produção do dispositivo fotosensor de infravermelho. Iniciando pela deposição das camadas ativas, passando pelo processamento do dispositivo e finalizando com a montagem do dispositivo em um sistema de leitura de dados.

Figura 3.2 - Tabela periódica com destaque em vermelho para as famílias componentes dos semicondutores III-V. Destaque em azul para a coluna onde se encontra presente o silício que será utilizado como dopante $\mathrm{n}$, entrando no lugar dos átomos do grupo III na rede cristalina. 
Figura 3.3 - Esquematização simplificada do funcionamento de um reator de MOVPE para crescimento de semicondutores da família III-V. Scrubber é o sistema de lavamento dos gases tóxicos na saida da câmara.

Figura 3.4 - Figura qualitativa sobre o comportamento das moléculas metalorgânicas ou hidretos quando atingem a região da câmara onde está localizado o substrato. As moléculas são quebradas devido à alta temperatura do substrato e os radicais hidrogênio e carbono são expelidos em forma de gás, enquanto os átomos metálicos difundem sobre a superfície em busca de um sítio de energia mínima.

Figura 3.5 - Gráfico de energia de gap em função do parâmetro de rede de semicondutores III-V. Repare que o InP possui um parâmetro de rede de 5,8688 angstrom [23], e as ligas de InGaAs e InAlAs possuem apenas uma composição possível que satisfaz esse parâmetro.

Figura 3.6 - Representação da célula unitária de um composto do tipo zinc-blende. Trata-se de uma rede FCC do material A com o material B inserido a $1 / 4$ das 4 diagonais principais do cubo.

Figura 3.7 - A difração de raios-X de comprimento de onda 1 por uma amostra com planos interatômicos separados por d.

Figura 3.8 - Experimento de difração de raios-X utilizando-se um cristal de referência com intuito de estreitar a dispersão em comprimento de onda do feixe emitido pela fonte de raios-X.

Figura 3.9 - Exemplo de um experimento de difração de raios-X. Temos a intensidade de espalhamento do raio- $X$ incidente sobre a amostra em função do ângulo espalhado. $O$ pico maior normalmente está relacionado com a difração do substrato dado a sua maior espessura, enquanto que o segundo pico está relacionado com a camada depositada.

Figura 3.10 - Exemplo do surgimento das franjas de Pendellosung para uma camada descasada com o substrato. 
Figura 3.11 - Exlicação gráfica de um experimento de fotoluminescência convencional.

Figura 3.12 - Representação esquemática do experimento de fotoluminescência utilizado. O laser, em verde, é modulado e passa por um jogo de espelhos e um jogo de lentes para que seja focado sobre a amostra. A emissão da amostra, em vermelho, também passa por um jogo de lentes para ser focada dentro do monocromador.

Figura 3.13 - Representação do experimento de efeito Hall. Nos contatos diametralmente opostos (1 e 3 ou 2 e 4 ) introduz-se uma corrente elétrica sob ação do campo magnético B. Nos 2 contatos restantes mede-se a voltagem Hall resultante.

Figura 3.14 - Passo a passo do processo de preparação da amostra para o experimento de efeito Hall.

Figura 3.15 - Foto do equipamento de efeito Hall da BIO RAD.

Figura 3.16 - Direção de propagação da corrente elétrica gerada pela radiação infravermelha dentro do dispositivo.

Figura 3.17 - Fotografia da alinhadora de máscaras localizada no LabSem / CETUC / PUC-Rio.

Figura 3.18 - Esquematização do processo de fotolitografia seguida de corrosão. Primeiramente é realizado o spin coating do fotoresiste sobre a amostra, o padrão é impresso e o conjunto é mergulhado na solução ácida pelo tempo estimado.

Figura 3.19 - Fotografia do perfilômetro por sonda. E uma figura esquemática do funcionamento do equipamento que mapeia a topografia da amostra através de uma sonda fina.

Figura 3.20 - Esquematização do processo de litografia seguida de metalização. Inicia-se imprimindo o padrão em resina sobre a amostra já corroída, realiza-se a evaporação de metal sobre a superfície da amostra e, por fim, retira-se o metal que foi depositado sobre a fotoresina, mantendo-se apenas o metal que foi depositado diretamente sobre o semicondutor. 
Figura 3.21 - Fotografia de uma amostra feita para teste de TLM. O padrão apresentado possui 9 pads distanciados de valores conhecidos.

Figura 3.22 - Fotografia da evaporadora com um esquema de funcionamento da evaporação do metal.

Figura 3.23 - Representação do dispositivo antes do polimento (à esquerda) e após o polimento à $45^{\circ}$ (à direita).

Figura 3.24 - Fotografia do experimento com todos os elementos necessários. No desenho à direita temos uma representação mais simplificada do processo.

Figura 3.25 - Desenho da grade de difração produzida sobre a amostra.

Figura 3.26 - Fotografia do processo de polimento com solução de bromo-metanol.

Figura 3.27 - Desenho pictográfico da integração do dispositivo processado sobre um suporte para leitura do sinal de corrente.

Figura 3.28 - Esquematização do processo de passivação, muito similar ao processo de fotolitografia. Neste caso como se trata da última etapa, há a necessidade de um alinhamento por sobre o padrão já impresso sobre a amostra.

Figura 3.29 - Fotografia do equipamento de corte das amostras e dos suportes. Localizado no CTI em Campinas.

Figura 3.30 - Esquematização do processo de alinhamento da amostra sobre a alumina a fim de soldar os contatos elétricos. Temos também representado o fio de ouro soldado sobre um dos pads do suporte de alumina.

Figura 3.31 - Fotografia da microsoldadora Ball Bonder de fio de ouro. $\mathrm{Na}$ figura 3.21 temos a foto de uma amostra onde foram realizadas as soldas com este equipamento em pads de $100 \times 100$ micra.

Figura 3.32 - Fotografia do equipamento de medida de corrente em função da voltagem aplicada. A montagem acima é feita para uso com headers comerciais.

Figura 3.33 - Montagem de medição de corrente contra voltagem sem a necessidade da utilização de um header. 
Figura 3.34 - Exemplo da curva IV de um fotodiodo de InGaAs. Temos representadas a resistência convencional $\mathrm{R}$ dado por $\mathrm{V} / \mathrm{I}$, e também a resistência dinâmica $r$ dado pela derivada da curva IV em um dado ponto.

Figura 3.35 - Esquematização do experimento de fotocorrente com um sistema utilizando uma fonte de emissão larga acoplada com um monocromador.

Figura 3.36 - Esquematização do experimento de fotocorrente utilizando um espectrômetro da Thermo Nicolet. Em destaque temos a representação de um interferômetro de Michelson que é utilizado no equipamento.

Figura 3.37 - Esquema de medição da responsividade de corpo negro para uma amostra a $77 \mathrm{~K}$.

Figura 3.38 - Fotografia da montagem experimental de responsividade de corpo negro no INPE localizado em São José dos Campos. [19]

Figura 3.39 - Gráfico de $\mathrm{Id}^{2}$ em função de d para obtenção da constante utilizada no cálcula da responsividade de pico.

Figura 3.40 - Gráfico de um exemplo de integração numérica de $\mathrm{M}(\mathrm{T}, 1) . \mathrm{s}(1)$.

Figura 4.1 - Gráfico empírico para calibração do composto de InAlAs sobre um substrato de InP.

Figura 4.2 - Gráfico empírico para calibração do composto de InGaAs sobre um substrato de InP.

Figura 4.3 - Gráfico da calibração da taxa de crescimento para InGaAs e InAlAs. Reparem que durante o histórico do laboratório estudouse diversas condições de fluxo do grupo III e de temperaturas distintas.

Figura 4.4 - Gráfico de fotoluminescência para uma amostra de InAlAs casada com InP.

Figura 4.5 - Gráfico de fotoluminescência para uma amostra de InGaAs casada com InP. 
Figura 4.6 - Curva de calibração da dopagem para InGaAs dopado com silício. Note que há uma tendência a saturação para valores altos de fluxo de silana.

Figura 4.7 - Esquematização dos valores nominais de espessura do dispositivo final. As camadas InGaAs:Si dos extremos são as camadas de contato. O restante é a camada ativa de poços quânticos do dispositivo.

Figura 4.8 - Resultado da difração de raios-X pelo dispositivo final. Presença de pico único e franjas bem definidas.

Figura 4.9 - Simulação de um poço de potencial de InGaAs com barreiras de InAlAs para temperaturas baixas.

Figura 4.10 - Luminescência em função do comprimento de onda para diversas temperaturas.

Figura 4.11 - Comportamento da energia do pico de luminescência em função da temperatura.

Figura 4.12 - Foto do processamento da amostra que será utilizada para as medidas em chanfro e em grade de difração produzida por FIB. Repare que há diversas texturizações distintas para que possamos obter a grade mais adequada para acoplamento da radiação.

Figura 4.13 - Foto das amostras nas dimensões corretas com grade feita por laser writer. Repare que as metalizações dos contatos inferior e superior estão espaçadas.

Figura 4.14 - Microscopia eletrônica de varredura da mesa. Repare nas grades de difração produzidas por FIB sobre as mesas. Temos presentes 4 texturizações distintas.

Figura 4.15 - Microscopia eletrônica de varredura da grade de difração produzida por FIB.

Figura 4.16 - Microscopia de força atômica da grade de difração produzida por laser writer.

Figura 4.17 - Fotografia de uma amostrada montada em um header polida a $45^{\circ}$.

Figura 4.18 - Montagem das amostras de chanfro e de grade de difração por FIB em headers comerciais modificados. 
Figura 4.19 - Resultado da medida de perfilometria sobre uma amostra processada e passivada. A região mais baixa dos gráficos na realidade correspondem ao topo da mesa, enquanto que as regiões mais altas corresponde à passivação.

Figura 4.20 - Gráfico de corrente de escuro para uma mesa passivada e uma mesa não passivada do mesmo processamento.

Figura 4.21 - Testes de aderência de diversas ligas metálicas sobre a mesa com intuito de buscar a melhor solda para o dispositivo final.

Figura 4.22 - Fotografia da amostra com grade de difração feita por laser writer completamente montada e pronta para ser medida.

Figura 4.23 - As amostras utilizadas para as medidas de caracterização.

Figura 4.24 - Corrente de escuro em função da temperatura para amostra de grade por laser writer.

Figura 4.25 - Gráfico para cálculo da energia de ativação para altas temperaturas em função da voltagem aplicada para amostra de grade por laser writer.

Figura 4.26 - Gráfico para cálculo da energia de ativação para altas temperaturas em função da voltagem aplicada para a amostra da referência [2].

Figura 4.27 - Corrente de escuro a 300K em função da área da mesa para diversas voltagens aplicadas. A amostra com $0,01 \mathrm{~mm}^{2}$ de área é a mesa com grade por FIB, a de $0,1 \mathrm{~mm}^{2}$ é a mesa com grade por laser writer, e a de $0,6 \mathrm{~mm}^{2}$ é a mesa da referência [2].

Figura 4.28 - Gráfico da resistência dinâmica do ponto zero em função da temperatura. A resistência varia de 104 até 107 ohms.

Figura 4.29 - Espectro de fotocorrente a $77 \mathrm{~K}$ em função da voltagem aplicada. Os valores negativos de fotocorrente foram refletidas pois o experimento fornece apenas o valor absoluto sem distinção de sentido.

Figura 4.30 - Comportamento da amplitude do pico de fotocorrente em função da voltagem aplicada sobre o dispositivo. Repare em uma tendência a saturação em $\pm 5 \mathrm{~V}$. 
Figura 4.31 - Comportamento da largura a meia altura (FWHM) do pico de fotocorrente em função da voltagem aplicada sobre o dispositivo.

Figura 4.32 - Espectro de fotocorrente a $45 \mathrm{~K}$ em função da voltagem aplicada. Os valores negativos de fotocorrente foram refletidos, pois o experimento fornece apenas o valor absoluto sem distinção de sentido.

Figura 4.33 - Gráfico de fotocorrente normalizado em função da voltagem aplicada para a amostra de grade por laser writer a $77 \mathrm{~K}$.

Figura 4.34 - Banda de condução do QWIP com a representação gráfica do estado fundamental (N1) e das bandas virtuais de energia no contínuo (B\#) calculadas.

Figura 4.35 - Gráfico de $\ln \left(\mathrm{Id}^{2}\right)$ em função de d para estimar a absorção atmosférica efetiva. Utilizamos um exemplo de medidas feitas para a amostra com grade por FIB.

Figura 4.36 - Resultado de responsividade de corpo negro para amostras com grade de difração e chanfro ambas a $77 \mathrm{~K}$ e com corpo negro a 900K (pico de emissão em 3,2 micra). Text02 e Text04 representam grades onde $a=b=640 \mathrm{~nm}$. Text06 representa grade onde $a=910 \mathrm{~nm}$ e $b=380 \mathrm{~nm}$. Os 3 casos possuem $\mathrm{h}=320 \mathrm{~nm}$.

Figura 4.37 - Gráfico de $\operatorname{Id}^{2}$ em função da distância para atestar que não temos presente a absorção atmosférica e para ser utilizado no cálculo da responsividade espectral.

Figura 4.38 - Comportamento da responsividade das amostras com grade por laser writer em função da voltagem aplicada para um corpo negro a 500K (pico de emissão em 5,8 micra).

Figura 4.39 - Comportamento da responsividade das amostras com grade por laser writer em função da voltagem aplicada para um corpo negro a 700K (pico de emissão em 4,1 micra).

Figura 4.40 - Responsividade espectral da amostra de grade feita com laser writer. Vemos um sinal mais intenso para voltagens negativas. 
Figura 4.41 - Detectividade integral da amostra com grade produzida por laser writer para um corpo negro a 500K. Não há uma simetria como nos resultados de responsividade, contudo a preferência pelas voltagens negativas se mantém.

Figura 4.42 - Detectividade integral da amostra com grade produzida por laser writer para um corpo negro a $700 \mathrm{~K}$. Assim como para 500K não há uma simetria mas há a preferência pelas voltagens negativas.

Figura 4.43 - Detectividade espectral para a amostra de grade produzida por laser writer para voltagens positivas.

Figura 4.44 - Detectividade espectral para a amostra de grade produzida por laser writer para voltagens negativas.

Figura A1 - Corrente gerada com o aumento da potência de radiação incidente sobre a face processada. Sobre a face do substrato a potência luminosa do equipamento de FTIR se mantém constante. À temperatura era controlada a 77K.

Figura A2 - Espectro de fotocorrente em função da potência de luz incidente pela face processada a temperatura aproximada de $77 \mathrm{~K}$.

Figura A3 - Espectro de fotocorrente em função da potência de luz incidente pela face processada. Refeito de forma detalhada para potências mais baixas da luz. Melhor controle de temperatura a $77 \mathrm{~K}$.

Figura A4 - Comportamento do pico de fotocorrente em função da potência de luz incidente. Repare que o crescimento da fotocorrente é alta para valores abaixo de 10 watts e depois se mantém relativamente estável até altas potências. A instabilidade se deve ao controle pouco efeitivo da temperatura para altas potências de luz.

Figura A5 - Comportamento do pico de fotocorrente em função da potência de luz incidente detalhado para baixas potências. Repare que neste caso há estabilidade e podemos estimar que a saturação ocorre para potência em torno de 5 a 6 watts. 


\section{Lista de tabelas}

Tabela 1.1 - Nomenclaturas da classificação do infravermelho utilizadas neste trabalho. [4]

Tabela 1.2 - Exemplos de ignição espontânea para alguns materiais. Destaque para os materiais presentes em florestas que podem acarretar em incêndios naturais. Como veremos adiante, uma emissão em 3 micra corresponde a uma temperatura de aproximadamente $693^{\circ} \mathrm{C}$, enquanto que para 5 micra o correspondente em temperatura é $306^{\circ} \mathrm{C}$. 